Document downloaded from:

http://hdl.handle.net/10251/120544

This paper must be cited as:

Acedo Rodríguez, L.; Botella, M.; Cortés, J.; Hidalgo, J.; Maqueda, E.; Villanueva Micó, RJ. (2018). Swarm hybrid optimization for a piecewise model fitting applied to a glucose model. Journal of Systems and Information Technology. 20(4):9618-9627. https://doi.org/10.1108/JSIT-10-2017-0103

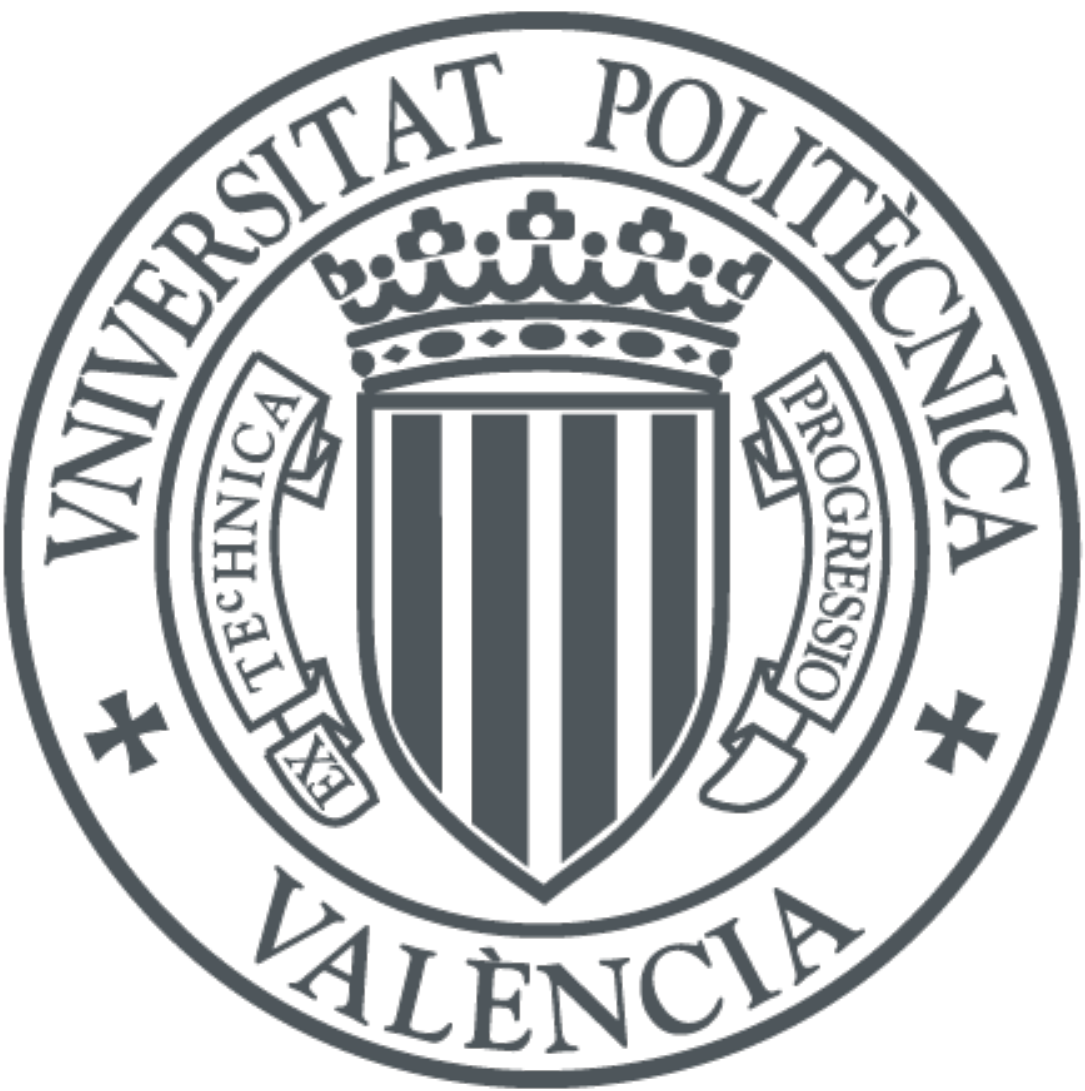

The final publication is available at

https://doi.org/10.1108/JSIT-10-2017-0103

Copyright Emerald

Additional Information 


\title{
Swarm hybrid optimization for a piecewise model fitting applied to a glucose model
}

\author{
L. Acedo, M. Botella, J.-C. Cortés, \\ J.-I. Hidalgo, E. Maqueda, R.-J. Villanueva
}

\begin{abstract}
Objective: Insulin pump therapy and accurate monitoring of glucose levels in diabetic patients are current research trends in diabetology. Both problems have a wide margin for improvement and promising applications in the control of parameters and levels involved. Methods: We have registered data for the levels of glucose in diabetic patients throughout a day with a temporal resolution of five minutes, the amount and time of insulin administered and time of ingestion. The estimated quantity of carbohydrates is also monitored. A mathematical model for Type 1 patients was fitted piecewise to this data and the evolution of the parameters was analyzed. Conclusion: We have found that the parameters for the model change abruptly throughout a day for the same patient, but this set of parameters account with precision for the evolution of the glucose levels in the test patients. This fitting technique could be used to personalize treatments for specific patients and predict the glucose levels variations in terms of hours or even shorter periods of time. This way more effective insulin pump therapies could be developed. Significance: The proposed model could allow for the development of improved schedules on insulin pumps therapies.
\end{abstract}

Index Terms-Glucose mathematical model, glucose monitoring, insulin pump therapy, piecewise fitting, swarm optimization

\section{INTRODUCTION}

Glucose real-time continuous monitoring (GRTCM) has proven a valid procedure to achieve metabolic control in Type 1 diabetic patients (DM1), both for multiple insulin dose treatment (MDI) and for continuous subcutaneous insulin infusion pump (ISCI) [1,2]. Using a combination of GRTCM and ISCI, with automated insulin suspension in response to hypoglycemia, the frequency and seriousness of hypoglycemia is greatly reduced [3-6].

L. Acedo, J. C. Cortés and R. J. Villanueva are with Instituto Universitario de Matemática Multidisciplinar, Building 8G, Access C, $2^{\circ}$ Floor, Universitat Politècnica de València, 46022, Camino de Vera s/n, Valencia, Spain. M. Botella works at Hospital Universitario Príncipe de Asturias, Alcalá de Henares, Madrid, Spain. J. I. Hidalgo is with the Universidad Complutense de Madrid, Madrid, Spain. E. Maqueda works at the Hospital Virgen de la Salud, Toledo, Spain. (correspondence e-mail: luiacrod@uoc.edu).
The main inconvenient of present GRTCM systems are their insufficient precision and reliability because these condition decision-making and they demand regular calibration with capillary glycemia autoanalysis [7].

On the other hand, the GRTCM patient must be instructed about continuous decision-making concerning the insulin doses to be administered in order to keep glycemic level within his/her therapeutic objectives, but for that the patient must take into account not only the present value of glycemic level but also the trend in the last few hours.

The magnitude of glycemic decrease after administering one unit of insulin (insulin sensitivity) is one of the key factors to be taken into account by professionals and patients to decide the insulin doses to be administered. Insulin sensitivity suffers from large inter and intraindividual variability, being conditioned by many factors such as: age, sex, weight, exercise, metabolic stress, meals, time of the day, etc, in such a way that it is very difficult for the patient to make correct decisions. All these factors give us a sufficient reason to improve the application of current mathematical models to diabetic patients.

For these reasons it would be interesting to develop a mathematical model capable of fitting the data for glucose levels in a patient and to make predictions under certain conditions that could gauge the risk of hypoglycaemia and the necessity of insulin administration along the day. This model should be applied and calibrated for every patient in particular because the physiological response can be very different from one patient to another.

Mathematical modelling of diabetes has focused in different aspects of the disease and it is already an active field of research [8-10]. Glucose metabolism coupled to insulin secretion has also been simulated with several models [11] as well as the pathogenesis of the disease [12]. Other authors have modelled the intravenous glucose tolerance test and the glycemic index as markers characterizing the response of the patient to the ingestion of food and sugar metabolism [13-16]. The progression of the disease and the deterioration of betacells' function has also been the topic of several models [1719]. 
Here we will use a mathematical model based upon differential equations as proposed by Prud homme et al. [20]. This model was developed with the objective of achieving control of postprandial glucose levels and it contains six unknown parameters related to the bioavailability of the meal, the insulin absorption and sensitivity, and the endogenous glucose production and effectiveness at zero insulin. Instead of using the system in Ref. [20] we will rewrite it in terms of difference equations with the objective of implementing it in a numerical scheme.

Our objective is to apply this model to obtain and accurate fitting of the evolution of glucose concentrations in particular individuals. This is achieved by a piecewise fitting with the temporal resolution of the measurements performed by the sensor. We will use a hybrid optimization technique which merges heuristic (Particle Swarm Optimization, PSO) [22] and deterministic methods (Nelder-Mead optimization) [23]. This way a very precise fitting is obtained and information about the fast evolution of the model parameters is also obtained.

Another alternative is to use global parameters for the whole period of time of interest (one day in the case of diabetic patients) but this is usually very ineffective for complex biological signals. As an example of this drawback, we have recently shown that the epidemiological data for varicella can only be fitted piecewise by obtaining a time-dependent seasonal forcing [21].

In this paper we show how to obtain model parameters for diabetic patients that could be used to assess the insulin dosing and to perform better control of their disease.

\section{METHODS}

In order to control the glucose, diabetics need to decide the insulin dose to be injected having into consideration food intakes, exercise done, etc, and try to predict the blood glucose level to avoid hypoglycemia (which can be a very dangerous situation). To help with this task, some models to measure and predict the levels of glucose in the human body have been presented, most of them are averaged models [8].

One of the main issues on the identification of models is the high variability of the glucose profiles from one patient to another. Thus, nowadays, the mainstream consists of customizing the treatments to every patient. With this aim, we use the model presented in [20] to be applied to a real patient. Although [20] presents a minimal model, we can characterize it for each patient, and the parameters of the model can be associated to values used in the daily therapy. We can take advantage of modern handy devices Continues Glucose Monitoring Systems (CGMS) that provides glucose measures (with some errors) every 5 minutes.

In this work we performed measurements with a CGMS every five minutes for a selected patient. A particular example, to be used in our model, is plotted in Fig. 1.
The intake of carbohydrates has been done at 08:10 and 14:22 in quantities of 50 grams and 60 grams, respectively. The levels of insulin injected can be seen in Fig. 2.

\section{A. Mathematical Model}

We are going to consider a discrete and adapted version of the model presented in [20], given by the following system of non-linear difference equations (some details concerning the rewriting of Eq. (3) are given at the Appendix):

$$
\begin{aligned}
& U_{t+1}=U_{t}+V_{t} \\
& V_{t+1}=V_{t}-2 a_{g} V_{t}-a_{g}^{2} U_{t}+K_{g} a_{g}^{2} C h_{t}, \\
& G_{t+1}=G_{t}-X_{t} G_{t}-S_{g 0} G_{t}+U_{\text {endo }}+C \frac{U_{t}}{M}, \\
& X_{t+1}=X_{t}-a_{x} X_{t}+a_{x} X_{t}^{1} \\
& X_{t+1}^{1}=X_{t}^{1}-a_{x} X_{t}^{1}+K_{x} a_{x} \frac{I_{t}}{M},
\end{aligned}
$$

where the variable parameters are defined as follows:

- $U_{t}$ is the gut glucose absorption at minute $t$ measured in $\mathrm{g} / \mathrm{min}$,

- $\quad V_{t}$ is the variation rate of the gut glucose absorption measured in $\mathrm{g} / \mathrm{min}^{2}$,

- $G_{t}$ is the level of glucose at minute $t$ measured in $\mathrm{mg} / \mathrm{dl}$,

- $X_{t}$ is the insulin action in $1 / \mathrm{min}$,

- $X_{t}^{1}$ is the intermediate insulin action measured also in $1 / \mathrm{min}$,

- $C h_{t}$ is the level of ingested carbohydrates at minute $t$ in $\mathrm{g} / \mathrm{min}$,

- $\quad I_{t}$ is the level of insulin injected at time $t$ measured in $\mathrm{mU} / \mathrm{min}$,

- $\quad C$ is a constant given by $50 / 9 \mathrm{mg} \mathrm{Kg/(dl} \mathrm{g),}$

- $a_{g}$ is the inverse of the meal time constant measured in $1 / \mathrm{min}$,

- $K_{g}$ is the unitless bioavailability of the meal of interest,

- $a_{x}$ is the inverse of the insulin absorption/action time constant measured in $1 / \mathrm{min}$,

- $K_{x}$ is the insulin sensitivity measured in $\mathrm{kg} / \mathrm{mU}$,

- $U_{\text {endo }}$ is the insulin independent endogenous glucose production measured in $\mathrm{mg} /(\mathrm{dl} \mathrm{min})$,

- $S_{g 0}$ is the glucose effectiveness at zero insulin measured in $1 / \mathrm{min}$,

- $\quad M$ is the weight of the patient in $\mathrm{kg},(\mathrm{M}=70$ for the current patient).

The quantities with the subscript $t$ are model functions for which we need initial conditions that should be known to integrate the model. In practice, only $G_{t}$ is measured at $\mathrm{t}=0$ but we will show how to obtain the rest of parameters by fitting. 


\section{B. Piecewise fitting}

For the records shown in Fig. 1 we can denote by $g_{5 t}, t=$ $1, \ldots, 96$, being the patient's glucose data every five minutes. With similar notation we have that $I_{t}, C_{t}, t=1, \ldots, 480$, are the injected insulin and ingested carbohydrates every minute, respectively. For our record we have $C h_{1}=50, C h_{373}=60$, and $C h_{t}=0$ for other time $t$.

The initial glucose level measured at 08:05 am is denoted by $g_{0}=102$ and this will be used as one of the initial conditions. It is convenient to cast the system of Eqs. (1)-(5) in a compact form:

$$
\mathbb{Y}_{t+1}=\mathbb{M}\left(\alpha ; \mathbb{Y}_{t}\right)
$$

where $\mathbb{Y}_{t}=\left(U_{t}, V_{t}, G_{t}, X_{t}, X_{t}^{1}\right)$ are the time-dependent quantities of the model and $\alpha$ stands for the unknown model parameters $\left(a_{x}, K_{x}, a_{g}, K_{g}, S_{g 0}, U_{\text {endo }}\right)$. The fitting process to be developed must cope with the problem arising from the fact that the only available data are the levels of glucose, $G_{t}$, measured every five minutes. The rest of quantities $U_{t}, V_{t}, X_{t}, X_{t}^{1}$ and the model parameters are unknowns. So, as our initial condition we will take:

$$
\mathbb{Y}_{0}=\left(A_{1}, A_{2}, g_{0}, A_{3}, A_{4}\right),
$$

where $A_{1}, \ldots, A_{4}$ are constants to be fitted and $g_{0}=102$.

An issue with the selection of these initial parameters is that, for some combination of them, the model predicts strong oscillations of the glucose levels. As we assume that in between of two subsequent measurements the behaviour of glucose dynamics is relatively smooth we will impose this smoothness as a condition in the initial parameter fitting.

The fitting procedure for the first iteration is then outlined as follows:

- STEP A: We perform a linear interpolation for the glucose levels at instants $\mathrm{t}=1$ minute, 2 minutes, 3 minutes and 4 minutes starting with the known data for the instants $\mathrm{t}=0$ and $\mathrm{t}=5$ minutes. This way we obtain the values: $f_{1}(t=0)=g_{0}, f_{1}(t=$ 1), $f_{1}(t=2), f_{1}(t=3), f_{1}(t=4), f_{1}(t=5)=$ $g_{5}$.

- STEP B: for a set of initial conditions as given in Eq. (7) we calculate the iterations:

$$
\mathbb{Y}_{1}=\mathbb{M}\left(\alpha ; \mathbb{Y}_{0}\right), \ldots \mathbb{Y}_{5}=\mathbb{M}\left(\alpha ; \mathbb{Y}_{4}\right)
$$

and from the third component of these vectors we get the prediction for the glucose levels: $G_{1}, G_{2}, G_{3}, G_{4}, G_{5}$.

- STEP C: we obtain the minimum for the absolute difference among the data and the prediction:

$$
\min \left\{\left|f_{1}(1)-G_{1}\right|, \ldots,\left|f_{1}(5)-G_{5}\right|\right\}
$$

Depending on this value and the best result until this iteration we choose another set of parameters $\left(a_{x}, K_{x}, a_{g}, K_{g}, S_{g 0}, U_{\text {endo }}\right)$ and $\mathbb{Y}_{5}$ as the new initial condition $\mathbb{Y}_{0}$. Then we go to STEP B again for a new prediction.

This procedure stops when the error is sufficiently small as described in the optimization procedure below.

\section{Swarm hybrid optimization}

Usually model fitting consists of minimizing certain objective functions to obtain the best set of parameters capable of making a consistent prediction for the experimental data. In our case we have in the i-th segment that the following quantity:

$\min \left\{\left|f_{i}(1)-G_{5(i-1)+1}\right|, \ldots,\left|f_{i}(5)-G_{5 i}\right|\right\}$

must be minimized. In optimization problems of this kind it is customary nowadays to use heuristic methods to perform an exploration of the parameter space instead of evaluating, systematically, the objective function for a large set of combinations of parameters. A widely used approach for heuristic optimization is the so-called Particle Swarm Optimization, PSO, method [22] in which the flight of a flock of birds is simulated to carry out a random exploration of parameter space to find the best values. If we are close to a good value it is, however, more efficient to use a deterministic method that converges faster to the best possible value in that region. So we have combined a PSO method with a NelderMead method [23] to achieve our optimization objective. Our combined optimization method proceeds in three general steps:

- STEP 1: We use a version of the Random Particle Swarm Optimization (rPSO) method [20] with mutation and random substitution of some birds in some steps. We used 40 birds to return the best position. In total we ran 1500 iterations for every five minutes interval of glucose measurements.

- STEP 2: As a second step we took the best position found in the previous step and build a small simplex around it as our initial guess for the Nelder-Mead algorithm [23]. The algorithm is run until the difference between two consecutive iterations is less than $10^{-6}$.

- STEP 3: We recover the parameter values for the best (minor error) result returned by the rPSO and the Nelder-Mead method. The result is accepted if its error is smaller than $5 \%$. Otherwise, we will start again the optimization until the error is acceptable. 


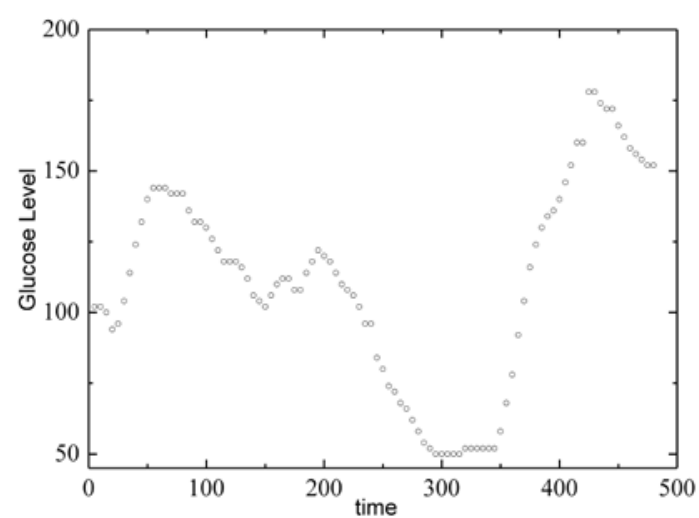

Fig. 1. Glucose levels of a diabetic patient taken every five minutes in a period from 8:06 am until 16:05 pm. The unit of concentration is $\mathrm{mg} / \mathrm{dl}$, time is given in minutes.

This upper value for the error is chosen that way because CGMS are designed with such maximum accuracy.

In some errors, however, the error is still larger than $5 \%$ after fifteen iterations of the hybrid optimization described above. This is, in particular, the case for the largest values of glucose levels in Fig. 1 around minute 425.

\section{RESULTS}

In Fig. 3 we can see that the resulting fitting for the glucose levels captures the details of the evolution throughout the eighth hours of monitored time. The errors as a function of time are depicted in Fig. 4, where we have achieved the objective of below $5 \%$ error values except for the interval around minute 425 . On the other hand, this does not affect the overall fitting of the model which is fairly good.

The model parameters are also shown in Fig. 5 for each five minutes interval. Notice that they show fast changes in short intervals of time pointing to a very volatile physiology of glucose and insulin in diabetic patients.

The other functions in the model of Eqs. (1)-(5) are indeed very regular and we predict an insulin action and intermediate insulin action, $\mathrm{X}_{\mathrm{t}}$ and $X_{t}^{1}$ which increase during the monitoring time for this patient.

Notice that the insulin action rises to a plateau at minute 300 to a value around $0.71 / \mathrm{min}$. This rise in insulin action approximately correlates with the second injection of insulin administered at 14:22 (corresponding to minute 376).

\section{CONCLUSIONS}

The combination of sensors and insulin pumps is every day more usual, almost standard, for the control of diabetic patients. Nevertheless, a poor understanding of the evolution of the blood glucose levels for given patients throughout the day is a major handicap for predicting the necessary amount of insulin to be delivered to the patient.

In this paper we have proposed a sequence of random and deterministic fitting methods to infer the model parameters for a recently proposed glucose model. We conclude that the parameters associated to the best fit of the glucose level for a patient monitored in a period of 8 hours fluctuate in short time intervals. Sometimes, even in a matter of minutes. This volatility is a manifestation of the natural instability of the insulin/glucose dynamics in diabetic patients.

Anyhow, we consider that a better statistical analysis of these variations could help us in predicting, in a personalized way, the amount of insulin necessary at each instant to avoid the chance of hypoglycemia and follow the schedules of the patient.

Work along this line is in progress and will be published elsewhere.

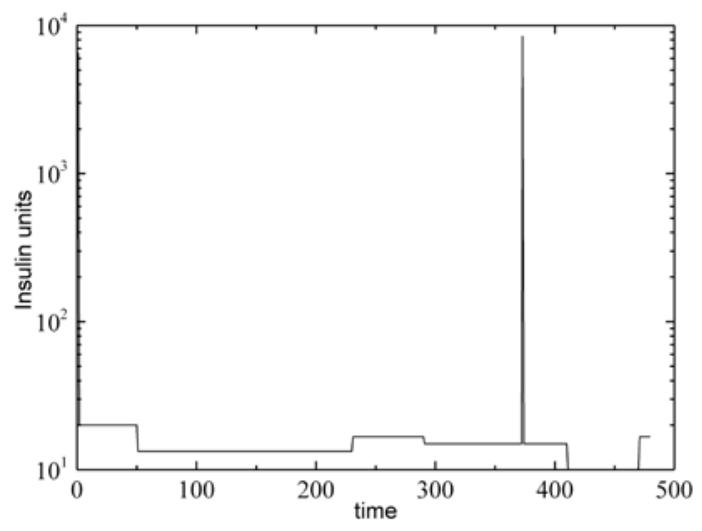

Fig. 2. Insulin units (Logarithmic scale) vs time for the monitored patient. 


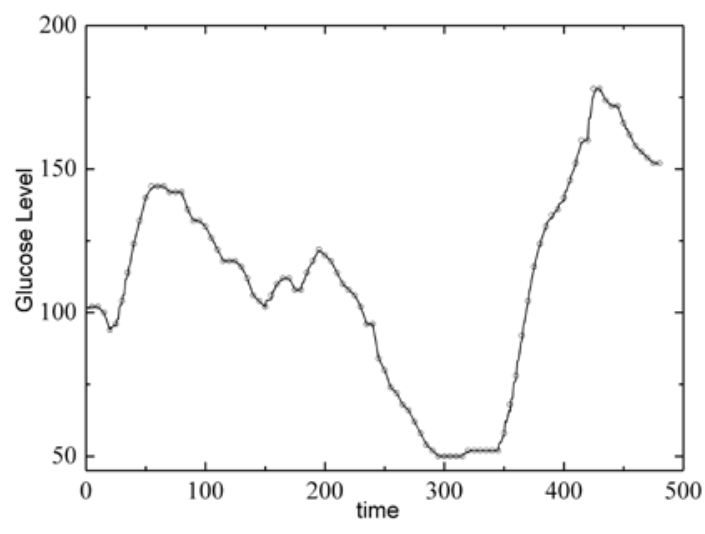

Fig 3. Observed levels of glucose (open circles) and result of the best fit (solid line) for the data in Fig. 1.

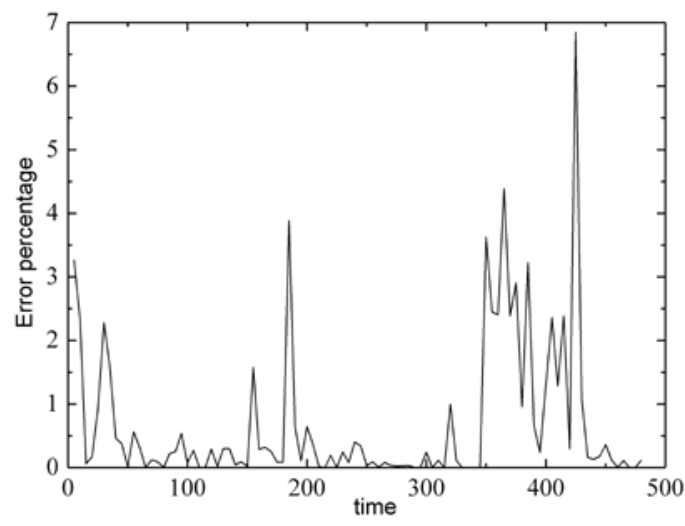

Fig 4. Errors in the fitted values of Fig. 3 as a function of time. Notice the high peak around minute 425 roughly corresponding to the sharp jump in glucose detected by the CGMS.

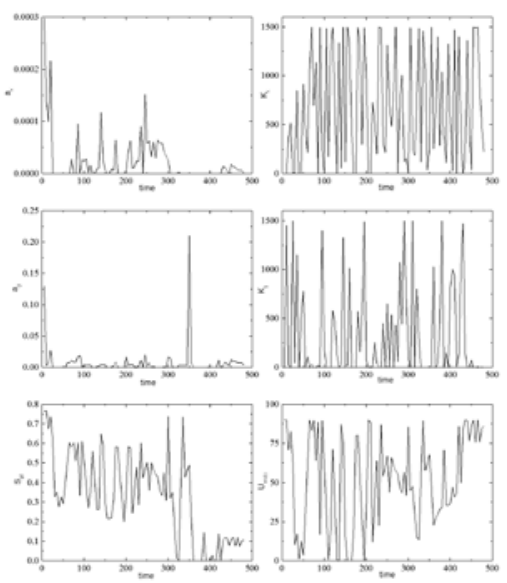

Fig 5. Parameters for the glucose model obtained for the best fit of the glucose levels: from top left to bottom right they are: $\mathrm{a}_{\mathrm{x}}, \mathrm{K}_{\mathrm{x}}, \mathrm{ag}_{\mathrm{g}}, \mathrm{K}_{\mathrm{g}}$, $\mathrm{s}_{\mathrm{g} 0}$ and $\mathrm{U}_{\text {endo }}$.

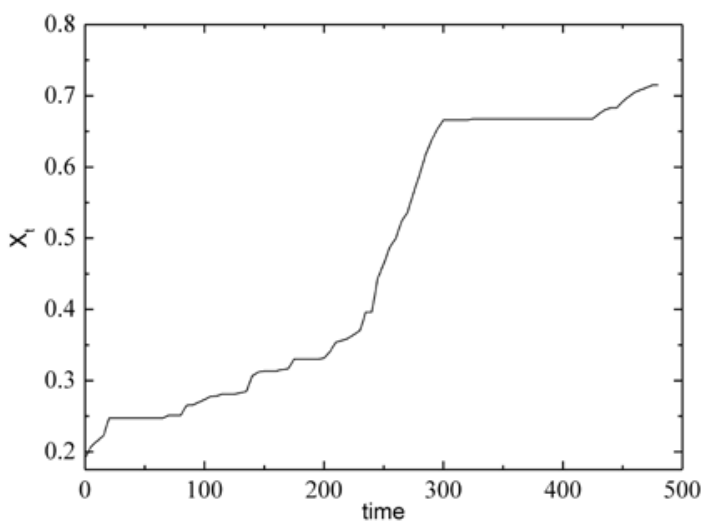

Fig 6. Insulin action in $1 /$ min vs time in minutes. The line is the prediction of the model.

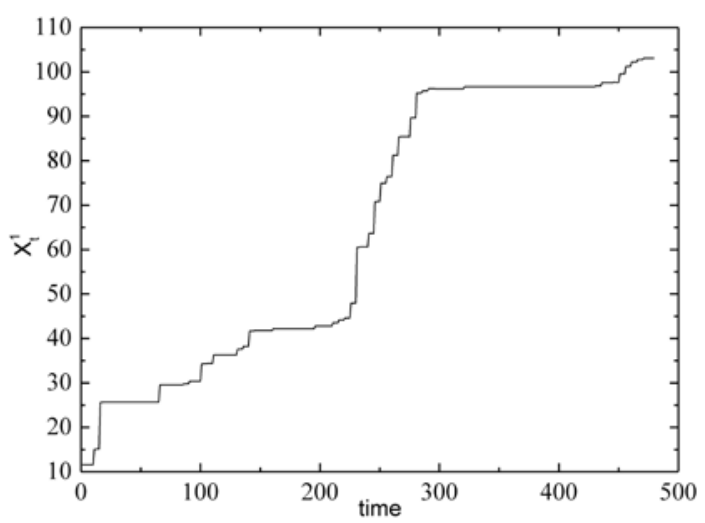

Fig 7. The intermediate insulin action in $1 /$ min vs time in minutes. The line is the prediction of the model.

\section{APPENDIX}

In this appendix we discuss the derivation of Eq. (3) in the discrete model we are using from the corresponding equation in the model of Prud'homme et al. [20]. In principle, we have in this reference that

$Q_{t+1}=Q_{t}-X_{t} Q_{t}-S_{g 0} Q_{t}+U_{\text {endo }}+C_{g \rightarrow m m o l} \frac{U_{t}}{M}$,

where $Q_{t}$ is the glucose amount in $\mathrm{mmol} / \mathrm{kg}$, and $U_{\text {endo }}$ is measured in $\mathrm{mmol} /(\mathrm{kg} \mathrm{min})$. But in our comparison with the CGMS's measurements we use instead the function $G_{t}$ whose 
units are mg/dl, i. e., milligrams of glucose per deciliter. Both quantities are related by:

$$
G_{t}=\frac{Q_{t}}{V_{g a}}
$$

where $V_{g a}$ is a conversion factor given as follows:

$$
1 \frac{m g}{d l}=\frac{1}{V_{g a}} \frac{m m o l}{k g}
$$

The conversion factor $C_{g \rightarrow \text { mmol }}$ from grams of glucose to mmol is obtained from the molecular weight of glucose, i.e., $180 \mathrm{~g} / \mathrm{mol}$ which implies $C_{g \rightarrow \text { mmol }}=\frac{50}{9} \mathrm{mmol} / \mathrm{g}$,

Then, if we divide all terms in Eq. (11) by $V_{g a}$ we obtain the evolution equation for $G_{t}$ as follows:

$$
G_{t+1}=G_{t}-X_{t} G_{t}-S_{g 0} G_{t}+\widehat{U}_{e n d o}+C \frac{U_{t}}{M}
$$

where $\widehat{U}_{\text {endo }}$ is the production of endogenous glucose measured in $\mathrm{mmol} /(\mathrm{kg} \mathrm{min})$ and $\mathrm{C}$ is a constant:

$$
C=\frac{50}{9} \frac{m g K g}{d l g}
$$

Eqs. (14) and (15) correspond to Eq. (5) in the form used in this paper.

\section{REFERENCES}

[1] M. Langendam, Y. M. Luijf, L. Hooft, J. H. DeVries, A. H. Mudde, R. J. Scholten, Continuous glucose monitoring systems for type 1 diabetes mellitus (Review), Cochrane Database Syst. Rev. 18(1) (2012) CD008101.

[2] J. C. Wong, N. C. Foster, D. M. Maahs, D. Raghinaru, R. M. Bergenstal, A. J. Ahmann et al., Real-Time Continuous Glucose Monitoring Among Participants in the T1D Exchange Clinic Registry, Diabetes Care 37 (2014) 2702-09.

[3] R. M. Bergenstal, W. V. Tamborlane, A. Ahmann, J. B. Buse, G. Dailey, S. N. Davis, Effectiveness of sensor-augmented insulin-pump therapy in type 1 diabetes, N. Engl. J. Med. 363 (2010) 311-20.

[4] T. T. Ly, J. A. Nicholas, A. Retterath, E. M. Lim, E. A. Davis, T. W. Jones, Effect of sensor-augmented insulin pump therapy and automated insulin suspension vs. standard insulin pump therapy on hypoglycemia in patients with type 1 diabetes: a randomized clinical trial, JAMA 310 (2013) 1240-47.

[5] P. Choudhary, S. Ramasamy, L. Green, G. Gallen, S. Pender, A. Brackenridge et al. Real-time continuous glucose monitoring significantly reduces severe hypoglycemia in hypoglycemia-unaware patients with type 1 diabetes. Diabetes Care 36 (2013) 4160-62.

[6] S. Garg, R. L. Brazg, T. S. Bailey, B. A. Buckingham, R. H. Slover, D. C. Klonoff et al., Reduction in duration of hypoglycemia by automatic suspension of insulin delivery: The In-Clinic ASPIRE Study, Diabetes Technol. Ther. 14 (2012) 205-9.

[7] W. H. Polonsky, D. Hessler, Perceived accuracy in continuous glucose monitoring: understand the impact on patients, J. Diabetes Sci. Technol. 9 (2015) 339-41
[8] A. Boutayeb, A. Chetouani, A critical review of mathematical models and data used in diabetology, Biomed. Eng. Online 5 (43) (2006).

[9] A. Makroglou, J. Li, Y. Kuang, Mathematical models and software tools for the glucose-insulin regulatory system and diabetes: an overview, Applied Numerical Mathematics 56 (2006) 559-573.

[10] C. B. Landersdorfer, W. J. Jusko, Pharmacokinetic/pharmacodynamic modelling in diabetes mellitus, Clinical pharmacokinetics 47(7) (2008) 41748.

[11] A. Mari, Mathematical modelling in glucose metabolism and insulin secretion, Current Opinion Clinical Nutrition Metabolism Care 5 (2002) 495501.

[12] R. N. Bergman, Pathogenesis and prediction of diabetes mellitus: lessons from integrative physiology, Mount Sinai J. Medicine 60 (2002) 280-290.

[13] A. De Gaetano, O. Arino, Mathematical Modelling of the Intravenous Glucose Tolerance Test, J. Math. Biol. 40 (2000) 136-168.

[14] M. Kalergis, E. De Grandpre, C. Andersons, The Role of the Glycemic Index in the Prevention and Management of Diabetes: A Review and Discussion, Canadian Journal of Diabetes 29(1) (2005) 27-38.

[15] A. Mukhopadhyay, A. De Gaetano, O. Arino, Modelling the intravenous glucose tolerance test: A global study for single-distributeddelay model, Discrete and Continous Dynamical Systems Series B 4(2) (2004) 407-417.

[16] C. L. Rohlfing, H. M. Wiedmeyer, R. R. Little, J. D. England, A. Tennill, D. E. Goldstein, Defining the relationship between plasma glucose and HbAlc: analysis of glucose profiles and HbAlc in the Diabetes Control and Complications Trial, Diabetes Care 25 (2000) 275-278.

[17] D. R. Matthews, J. P. Hosker, A. S. Rudenski et al., Homeostasis model assessment: insulin resistance and beta cell function from fasting plasma glucose and insulin concentrations in man, Diabetologia 28 (1985) 412-419.

[18] J. C. Levy, D. R. Matthews, M. P. Hermans, Correct Homeostasis Model Assessment (HOMA) evaluation uses the computer program, Diabetes Care 20 (1998) 2191-2192.

[19] A. Bagust, S. Beale, Deteriorating beta-cell function in type 2 diabetes: a long-term model, Q. J. Med. 96 (2003) 281-288.

[20] T. Prud'homme, A. Bock, G. François et al., Preclinically assessed optimal control of postprandial glucose excursions for type 1 patients with diabetes, IEEE Conference on Automation Science and Engineering (CASE), 2011, DOI: 10.1109/CASE.2011.6042510

[21] L. Acedo, J. A. Moraño, F. J. Santonja et al., A deterministic model for highly contagious diseases: The case of varicella, Physica A: Statistical Mechanics and its Applications 450 (2016) 278-286.

[22] C. Jacob, N. Khemka, Particle Swarm Optimization in Mathematica. An Exploration Kit for Evolutionary Optimization, IMS'04, Proc. Sixth International Mathematica Symposium, Banff, Canada, (2004).

[23] J. A. Nelder, R. Mead, A simplex method for function minimization, Computer Journal 7 (1964) 308-313. 